PROCEEDINGS OF THE

AMERICAN MATHEMATICAL SOCIETY

Volume 134, Number 12, December 2006, Pages 3417-3421

S 0002-9939(06)08379-1

Article electronically published on May 31, 2006

\title{
SYMMETRIC MODULAR DIOPHANTINE INEQUALITIES
}

\author{
J. C. ROSALES
}

(Communicated by Lance W. Small)

\begin{abstract}
In this paper we study and characterize those Diophantine inequalities $a x \bmod b \leq x$ whose set of solutions is a symmetric numerical semigroup.
\end{abstract}

Given two integers $a$ and $b$ with $b \neq 0$ we write $a \bmod b$ to denote the remainder of the division of $a$ by $b$. Following the notation used in [8], a modular Diophantine inequality is an expression of the form $a x \bmod b \leq x$. The set $\mathrm{S}(a, b)$ of integer solutions of this inequality is a numerical semigroup, that is, it is a subset of $\mathbb{N}$ (the set of nonnegative integers) closed under addition, $0 \in \mathrm{S}(a, b)$ and such that $\mathbb{N} \backslash \mathrm{S}(a, b)$ has finitely many elements. We say that a numerical semigroup is modular if it is the set of solutions to a modular Diophantine inequality. As shown in 8], not every numerical semigroup is of this form.

If $S$ is a numerical semigroup, then the greatest integer not in $S$ is the Frobenius number of $S$, denoted by $\mathrm{g}(S)$. The numerical semigroup $S$ is symmetric (see [1]) if $x \in \mathbb{Z} \backslash S$ implies $\mathrm{g}(S)-x \in S$ ( $\mathbb{Z}$ is the set of integers). This kind of semigroup has been widely studied and characterized in the literature (see, for instance, [2, 4, 6]). We will say that the inequality $a x \bmod b \leq x$ is symmetric if $\mathrm{S}(a, b)$ is a symmetric numerical semigroup.

It is well known (see, for instance, 7]) that every numerical semigroup $S$ is finitely generated and thus there exist positive integers $n_{1}, \ldots, n_{p}$ such that

$$
S=\left\langle n_{1}, \ldots, n_{p}\right\rangle=\left\{a_{1} n_{1}+\cdots+a_{p} n_{p} \mid a_{1}, \ldots, a_{p} \in \mathbb{N}\right\} .
$$

If no proper subset of $\left\{n_{1}, \ldots, n_{p}\right\}$ generates $S$, then we say that this set is a minimal system of generators of $S$. Minimal systems of generators always exist and are unique (see [7]); the cardinality of a minimal system of generators of $S$ is known as the embedding dimension of $S$, denoted here by e $(S)$.

Clearly, the inequality $a x \bmod b \leq x$ has the same integer solutions as the inequality $(a \bmod b) x \bmod b \leq x$. Thus we may assume (and in fact we will) that $a, b \in \mathbb{N}$ and $a<b$. Note that $\mathrm{S}(0, b)=\mathbb{N}$ is trivially symmetric.

Throughout this paper (and unless otherwise stated) we will assume that $a$ and $b$ are positive integers, and that $d=\operatorname{gcd}\{a, b\}$ and $d^{\prime}=\operatorname{gcd}\{a-1, b\}$ (gcd stands for greatest common divisor). In Proposition 4 , we will show that $\mathrm{S}(a, b)$ is symmetric if and only if $\mathrm{S}(a, b)=\left\langle b / d, b / d^{\prime}, d+d^{\prime}\right\rangle$. Theorem 5 characterizes those pairs $(a, b)$

Received by the editors April 8, 2004 and, in revised form, June 21, 2005.

2000 Mathematics Subject Classification. Primary 20M14.

The author was supported by the project BFM2000-1469 and thanks P. A. García-Sánchez for his comments and suggestions. 
such that $\mathrm{S}(a, b)$ is symmetric. As a consequence, in Corollary [6 we show that $S$ is a modular symmetric numerical semigroup if and only if $S=\left\langle k t, k t^{\prime}, t+t^{\prime}\right\rangle$ where $k, t, t^{\prime}$ are positive integers such that $\operatorname{gcd}\left\{t, t^{\prime}\right\}=\operatorname{gcd}\left\{k, t+t^{\prime}\right\}=1$. If $S$ is a numerical semigroup with e $(S) \leq 2$, then by [5], $S$ is symmetric and by 9 we know that $S$ is modular. Proposition 9 states that $\mathrm{e}\left(\left\langle k t, k t^{\prime}, t+t^{\prime}\right\rangle\right)=3$ if and only if $1 \notin\left\{t, t^{\prime}, k\right\}$. We finish the paper proposing the open question of deciding for which positive integers $g$ there exists a modular symmetric numerical semigroup with embedding dimension three and Frobenius number $g$.

The following result is a consequence of Corollaries 6, 16 and 17, and Lemma 11 in [8]. Recall that $a$ and $b$ are positive integers, with $a<b$ and that $d=\operatorname{gcd}\{a, b\}$ and $d^{\prime}=\operatorname{gcd}\{a-1, b\}$.

Lemma 1. 1) If $x \in \mathbb{Z} \backslash \mathrm{S}(a, b)$, then $b-x \in \mathrm{S}(a, b)$.

2) Let $x$ be a positive integer. Then $x \in \mathrm{S}(a, b)$ and $b-x \in \mathrm{S}(a, b)$ if and only if

$$
x \in\left\{k \frac{b}{d} \mid 0 \leq k \leq d-1\right\} \cup\left\{k \frac{b}{d^{\prime}} \mid 0 \leq k \leq d^{\prime}-1\right\} .
$$

3) $b-d-d^{\prime} \geq \mathrm{g}(\mathrm{S}(a, b))$.

4) $\mathrm{S}(a, b)$ is symmetric if and only if $\mathrm{g}(\mathrm{S}(a, b))=b-d-d^{\prime}$.

The next result is broadly used throughout this paper.

Lemma 2. $\mathrm{S}(a, b)$ is symmetric if and only if $b-d-d^{\prime} \notin \mathrm{S}(a, b)$.

Proof. If $\mathrm{S}(a, b)$ is symmetric, then by 4$)$ in Lemma 1, we know that $\mathrm{g}(\mathrm{S}(a, b))=$ $b-d-d^{\prime}$ and thus $b-d-d^{\prime} \notin \mathrm{S}(a, b)$. Conversely, if $b-d-d^{\prime} \notin \mathrm{S}(a, b)$, then by 3$)$ in Lemma 1, we deduce that $\mathrm{g}(\mathrm{S}(a, b))=b-d-d^{\prime}$, which in view of 4$)$ in Lemma 1 implies that $\mathrm{S}(a, b)$ is symmetric.

In order to prove Proposition 4, we need the following result which can be deduced from $[5$.

Lemma 3. If $S=\left\langle n_{1}, n_{2}, n_{3}\right\rangle$ is a numerical semigroup and $\left(\operatorname{gcd}\left\{n_{1}, n_{2}\right\}\right) n_{3} \in$ $\left\langle n_{1}, n_{2}\right\rangle$, then $S$ is symmetric.

Proposition 4. $\mathrm{S}(a, b)$ is symmetric if and only if $\mathrm{S}(a, b)=\left\langle\frac{b}{d}, \frac{b}{d^{\prime}}, d+d^{\prime}\right\rangle$.

Proof. If $\mathrm{S}(a, b)$ is symmetric, then by Lemma 2 we know that $b-d-d^{\prime} \notin S$. Hence by 1) in Lemma 1, $d+d^{\prime} \in \mathrm{S}(a, b)$. Besides, $a \frac{b}{d} \bmod b=0$ and $a \frac{b}{d^{\prime}} \bmod b=$ $\left((a-1) \frac{b}{d^{\prime}}+\frac{b}{d^{\prime}}\right) \bmod b=\frac{b}{d^{\prime}} \bmod b \leq \frac{b}{d^{\prime}}$. Hence $\left\{\frac{b}{d}, \frac{b}{d^{\prime}}, d+d^{\prime}\right\} \subseteq \mathrm{S}(a, b)$. Consequently, $\left\langle\frac{b}{d}, \frac{b}{d^{\prime}}, d+d^{\prime}\right\rangle \subseteq \mathrm{S}(a, b)$. For the other inclusion, take $x \in \mathrm{S}(a, b)$ and let $t=\max \left\{k \in \mathbb{N} \mid x-k\left(d+d^{\prime}\right) \in \mathrm{S}(a, b)\right\}$. Then $x-(t+1)\left(d+d^{\prime}\right) \notin \mathrm{S}(a, b)$. By using that $\mathrm{S}(a, b)$ is symmetric and that $\mathrm{g}(\mathrm{S}(a, b))=b-d-d^{\prime}$, we obtain that $b-d-d^{\prime}-x+(t+1)\left(d+d^{\prime}\right)=b-\left(x-t\left(d+d^{\prime}\right)\right) \in \mathrm{S}(a, b)$. As $x-t\left(d+d^{\prime}\right)$ is also in $\mathrm{S}(a, b)$, by 2$)$ in Lemma 1 we conclude that $x \in\left\langle\frac{b}{d}, \frac{b}{d^{\prime}}, d+d^{\prime}\right\rangle$.

Conversely, since $\operatorname{gcd}\{a, a-1\}=1$, then $\operatorname{gcd}\left\{d, d^{\prime}\right\}=1$ and thus $\operatorname{gcd}\left\{\frac{b}{d}, \frac{b}{d^{\prime}}\right\}=$ $\frac{b}{d d^{\prime}}$. As $\frac{b}{d d^{\prime}}\left(d+d^{\prime}\right)=\frac{b}{d^{\prime}}+\frac{b}{d} \in\left\langle\frac{b}{d}, \frac{b}{d^{\prime}}\right\rangle$, by Lemma 3. we conclude that $\mathrm{S}(a, b)$ is symmetric.

Note that as an immediate consequence of this proposition, we have that if $\mathrm{S}(a, b)$ is symmetric, then $\mathrm{e}(\mathrm{S}(a, b)) \leq 3$. In view of [5], we deduce that if $S$ is a modular numerical semigroup, then $S$ is symmetric if and only if $S$ is a complete intersection; or equivalently, $S$ is free in the sense of [3].

Given two integers $x$ and $y$, denote by $x \mid y$ the fact that $x$ divides $y$. 
Theorem 5. $\mathrm{S}(a, b)$ is symmetric if and only if $(a, b)=\left(u t, k t t^{\prime}\right)$ for some positive integers $t, t^{\prime}, u, v$ and $k$ such that $u t-v t^{\prime}=1$ and $k \mid u+v$.

Proof. Necessity. Since $d=\operatorname{gcd}\{a, b\}$ and $d^{\prime}=\operatorname{gcd}\{a-1, b\}$, there exist positive integers $u, v$ and $k$ such that $a=u d, a-1=v d^{\prime}$ and $b=k d d^{\prime}$. Note that $u d-v d^{\prime}=1$ and $(a, b)=\left(u d, k d d^{\prime}\right)$. We show that if $\mathrm{S}(a, b)$ is symmetric, then $k \mid u+v$. If $k=1$, this assertion trivially holds. Thus, in the following we will assume that $k \geq 2$. We distinguish two cases depending on the value of $d^{\prime}$.

1) Assume that $d^{\prime}=1$. If $(1+u+v) \bmod k=0$, then $(u d)(d+1) \bmod k d=0$, since $(u d)(d+1) \bmod k d=(d(1+v)+u d) \bmod k d=d((1+u+v) \bmod k)=0$. Hence $u d(k d-(d+1)) \bmod k d=0$ and thus $k d-d-1 \in \mathrm{S}(u d, k d)$. But this is impossible, since $(u d, k d)=(a, b)$ and as $\mathrm{S}(a, b)$ is symmetric, by Lemma 2 $k d-d-1=b-d-d^{\prime} \notin \mathrm{S}(u d, k d)=\mathrm{S}(a, b)$. Therefore, $(1+u+v) \bmod k \neq 0$. By using the equalities seen above, $u d(k d-d-1) \bmod k d=k d-d((1+u+v) \bmod k)$. As $k d-d-1 \notin \mathrm{S}(u d, k d)$, we deduce that $k d-d((1+u+v) \bmod k)>k d-d-1$, whence $d+1>d((1+u+v) \bmod k)$. This implies that $(1+u+v) \bmod k=1$ and this leads to $(u+v) \bmod k=0$. This proves that $k \mid u+v$.

2) Assume now that $d^{\prime} \geq 2$. Note that

$$
\begin{aligned}
u d\left(d+d^{\prime}\right) \bmod k d d^{\prime} & =\left(d\left(1+v d^{\prime}\right)+u d d^{\prime}\right) \bmod k d d^{\prime} \\
& \left.=\left(d+(u+v) d d^{\prime}\right)\right) \bmod k d d^{\prime} \\
& =\left(d+d d^{\prime}((u+v) \bmod k)\right) \bmod k d d^{\prime} \\
& =d+d d^{\prime}((u+v) \bmod k) .
\end{aligned}
$$

The last equality holds because $d^{\prime} \geq 2$ and thus $d+d d^{\prime}((u+v) \bmod k) \leq$ $d+d d^{\prime}(k-1)<k d d^{\prime}$. Hence

$$
u d\left(k d d^{\prime}-d-d^{\prime}\right) \bmod k d d^{\prime}=k d d^{\prime}-d-d d^{\prime}((u+v) \bmod k) .
$$

As $\mathrm{S}\left(u d, k d d^{\prime}\right)=\mathrm{S}(a, b)$ is symmetric, by Lemma 2 we have that $k d d^{\prime}-d-d^{\prime} \notin$ $\mathrm{S}\left(u d, k d d^{\prime}\right)$ and consequently

$k d d^{\prime}-d-d d^{\prime}((u+v) \bmod k)=u d\left(k d d^{\prime}-d-d^{\prime}\right) \bmod k d d^{\prime}>k d d^{\prime}-d-d^{\prime}$.

This implies that $d^{\prime}>d d^{\prime}((u+v) \bmod k)$ and this forces $(u+v) \bmod k=0$. This proves that $k \mid u+v$ holds.

Sufficiency. Clearly, $\operatorname{gcd}\left\{u t,(u+v) t t^{\prime}\right\}=t$ and $\operatorname{gcd}\left\{u t-1,(u+v) t t^{\prime}\right\}=$ $\operatorname{gcd}\left\{v t^{\prime},(u+v) t t^{\prime}\right\}=t^{\prime}$. By using that $k$ divides $u+v$, we obtain that $\operatorname{gcd}\left\{u t, k t t^{\prime}\right\}=$ $t$ and $\operatorname{gcd}\left\{u t-1, k t t^{\prime}\right\}=t^{\prime}$. We apply Lemma2 2 to prove that $\mathrm{S}\left(u t, k t t^{\prime}\right)$ is symmetric. It suffices to show that $k t t^{\prime}-t-t^{\prime} \notin \mathrm{S}\left(u t, k t t^{\prime}\right)$. Note that for $k=t^{\prime}=1$, the result follows trivially, since $\mathrm{S}(u t, t)=\mathrm{S}(0, t)=\mathbb{N}$, which is symmetric. Thus, assume that $k \geq 2$ or $t^{\prime} \geq 2$. Note that $u t\left(t+t^{\prime}\right) \bmod k t t^{\prime}=\left(t\left(1+v t^{\prime}\right)+u t t^{\prime}\right) \bmod k t t^{\prime}=$ $\left(t+(u+v) t t^{\prime}\right) \bmod k t t^{\prime}=t$, since $k \mid u+v$ and $t<k t t^{\prime}$ because $k \geq 2$ or $t^{\prime} \geq 2$. Hence $u t\left(k t t^{\prime}-t-t^{\prime}\right) \bmod k t t^{\prime}=k t t^{\prime}-t>k t t^{\prime}-t-t^{\prime}$. This implies that $k t t^{\prime}-t-t^{\prime} \notin \mathrm{S}\left(u t, k t t^{\prime}\right)$ and this concludes the proof.

Corollary 6. Let $S$ be a numerical semigroup. Then $S$ is symmetric and modular if and only if $S=\left\langle k t, k t^{\prime}, t+t^{\prime}\right\rangle$, where $t, t^{\prime}$ and $k$ are positive integers such that $\operatorname{gcd}\left\{t, t^{\prime}\right\}=\operatorname{gcd}\left\{k, t+t^{\prime}\right\}=1$. Moreover, if this holds, then $\mathrm{g}(S)=k t t^{\prime}-t-t^{\prime}$.

Proof. If $S$ is a modular numerical semigroup, then there exist positive integers $a$ and $b$ such that $S=\mathrm{S}(a, b)$. If, in addition, $S$ is symmetric, then by Proposition 4 we 
have that $S=\mathrm{S}(a, b)=\left\langle\frac{b}{d}, \frac{b}{d^{\prime}}, d+d^{\prime}\right\rangle$, where $d=\operatorname{gcd}\{a, b\}$ and $d^{\prime}=\operatorname{gcd}\{a-1, b\}$. Note that $\operatorname{gcd}\left\{d, d^{\prime}\right\}=1$ and if $k=\frac{b}{d d^{\prime}}$, then $S=\left\langle k d, k d^{\prime}, d+d^{\prime}\right\rangle$. As $S$ is a numerical semigroup $\operatorname{gcd}\left\{k d, k d^{\prime}, d+d^{\prime}\right\}=1$, whence $\operatorname{gcd}\left\{k, d+d^{\prime}\right\}=1$.

Conversely, as $\operatorname{gcd}\left\{t, t^{\prime}\right\}=\operatorname{gcd}\left\{k, t+t^{\prime}\right\}=1$, we have that $\operatorname{gcd}\left\{k t, t+t^{\prime}\right\}=1$. Thus there exist positive integers $u, v$ such that $u k t-v\left(t+t^{\prime}\right)=1$, or equivalently $(u k-v) t-v t^{\prime}=1$. Besides, $k$ trivially divides $u k-v+v$. We can apply Theorem 5 and deduce that $\mathrm{S}\left((u k-v) t, k t t^{\prime}\right)$ is symmetric. Then in view of Proposition 4, we know that $\mathrm{S}\left((u k-v) t, k t t^{\prime}\right)=\left\langle k t, k t^{\prime}, t+t^{\prime}\right\rangle$ and by Lemma2, $\mathrm{g}(S)=k t t^{\prime}-t-t^{\prime}$.

Example 7. If we use Corollary 6 with $t=2, t^{\prime}=3$ and $k=7$, then $S:=\langle 14,21,5\rangle$ is a modular symmetric numerical semigroup with $\mathrm{g}(S)=37$. Moreover, from the proof of the above mentioned corollary, we can obtain a couple of positive integers $a, b$ such that $S=\mathrm{S}(a, b)$. It suffices to observe that $k t=14, t+t^{\prime}=5$ and $4 \times 14-11 \times 5=1$. Hence $u=4$ and $v=11$, which implies that $S=$ $\mathrm{S}\left((u k-v) t, k t t^{\prime}\right)=\mathrm{S}(34,42)=\langle 14,21,5\rangle$.

Example 8. $S:=\langle 6,7,9\rangle=\{0,6,7,9,12,13,14,15,16,18, \rightarrow\}$ (the arrow means that from this point on, every integer belongs to the set) is a symmetric numerical semigroup with $\mathrm{g}(S)=17$. By using Corollary [6 we can deduce that $S$ is not modular, since there are no positive integers $k, t$ and $t^{\prime}$ such that $\operatorname{gcd}\left\{t, t^{\prime}\right\}=$ $\operatorname{gcd}\left\{k, t+t^{\prime}\right\}=1$ and $\{6,7,9\}=\left\{k t, k t^{\prime}, t+t^{\prime}\right\}$.

Proposition 9. Let $k, t$ and $t^{\prime}$ be positive integers such that

$$
\operatorname{gcd}\left\{t, t^{\prime}\right\}=\operatorname{gcd}\left\{k, t+t^{\prime}\right\}=1 \text {. }
$$

Then $\mathrm{e}\left(\left\langle k t, k t^{\prime}, t+t^{\prime}\right\rangle\right)=3$ if and only if $1 \notin\left\{k, t, t^{\prime}\right\}$.

Proof. If $t=1$, then $\left\langle k, k t^{\prime}, t+t^{\prime}\right\rangle=\left\langle k, 1+t^{\prime}\right\rangle$. Thus $\mathrm{e}\left(\left\langle k t, k t^{\prime}, t+t^{\prime}\right\rangle\right) \leq 2$. The cases $t^{\prime}=1$ and $k=1$ are analogous.

Conversely, as $k \neq 1$, we have that $t+t^{\prime} \notin\left\langle k t, k t^{\prime}\right\rangle$, since otherwise $k$ would divide $t+t^{\prime}$, in contradiction with $\operatorname{gcd}\left\{k, t+t^{\prime}\right\}=1$. If $k t \in\left\langle k t^{\prime}, t+t^{\prime}\right\rangle$, then $k t=r k t^{\prime}+$ $s\left(t+t^{\prime}\right)$ for some nonnegative integers $r$ and $s$. The fact that $\operatorname{gcd}\left\{k, t+t^{\prime}\right\}=1$, implies that $k$ divides $s$ and thus we can write $t=r t^{\prime}+\frac{s}{k}\left(t+t^{\prime}\right)$. But this forces $s / k=0$ and $t=r t^{\prime}$, because $t, t^{\prime}>1$, contradicting that $\operatorname{gcd}\left\{t, t^{\prime}\right\}=1$. Analogously, one proves that $k t^{\prime} \notin\left\langle k t, t+t^{\prime}\right\rangle$. This shows that $\mathrm{e}\left(\left\langle k t, k t^{\prime}, t+t^{\prime}\right\rangle\right)=$ 3 .

It is well known (and easy to prove) that the Frobenius number of a symmetric numerical semigroup is always an odd integer. If $g$ is an odd positive integer, then in view of Corollary [6] with $t=t^{\prime}=1$ and $k=g+2$, we deduce that $\langle 2, g+2\rangle$ is a symmetric modular numerical semigroup with Frobenius number $g$ (this actually was already known to us prior to Corollary [6. since $\langle 2, g+2\rangle$ is symmetric with Frobenius number $g$ and it is modular in view of [9]). As we will see in Example 11, there is no symmetric modular numerical semigroup with embedding dimension three and Frobenius number nine. So the natural question arises: For which values of $g$ there exists a symmetric modular numerical semigroup with embedding dimension three and Frobenius number $g$ ? As a consequence of Corollary 6 and Proposition 9 we obtain the following result, which answers (though not in an effective way) the above question.

Corollary 10. Let $g$ be a positive integer. Then there exists a symmetric modular numerical semigroup with embedding dimension three and Frobenius number $g$ if 
and only if $g=k t t^{\prime}-t-t^{\prime}$ for some integers greater than or equal to two such that $\operatorname{gcd}\left\{t, t^{\prime}\right\}=\operatorname{gcd}\left\{k, t+t^{\prime}\right\}=1$.

Example 11. We prove that there are no symmetric modular numerical semigroups with embedding dimension three and Frobenius number nine. By using Corollary 10. it suffices to show that nine cannot be expressed as $k t t^{\prime}-t-t^{\prime}$ with $k, t, t^{\prime} \geq 2$ and $\operatorname{gcd}\left\{t, t^{\prime}\right\}=\operatorname{gcd}\left\{k, t+t^{\prime}\right\}=1$. As $t t^{\prime} \geq t+t^{\prime}$, if $9=k t t^{\prime}-t-t^{\prime}$, then $(k-1) t t^{\prime} \leq 9$. From the conditions imposed on $k, t$ and $t^{\prime}$, we deduce that $k=2$ and $\left\{t, t^{\prime}\right\}=\{2,3\}$. But then $k t t^{\prime}-t-t^{\prime}=7 \neq 9$.

We finish this work with an example where we give some families of positive integers $g$ for which there exists a symmetric modular numerical semigroup of embedding dimension three and Frobenius number $g$.

\section{Example 12.}

- If we apply Corollary 10 to $t=2$ and $t^{\prime}=3$, then we obtain that there exist symmetric modular numerical semigroups with embedding dimension three and Frobenius number $g$, for every $g$ in the set

$$
\{k 6-5 \mid k \geq 2, \operatorname{gcd}\{k, 5\}=1\}=\{7,13,19,31, \ldots\} .
$$

- For $t=2$ and $t^{\prime}=5$ we obtain the set

$$
\{k 10-7 \mid k \geq 2, \operatorname{gcd}\{k, 7\}=1\}=\{13,23,33,43,53,73, \ldots\} .
$$

- For $t=3$ and $t^{\prime}=4$ one gets the set

$$
\{k 12-7 \mid k \geq 2, \operatorname{gcd}\{k, 7\}=1\}=\{17,29,41,53,65,89, \ldots\} .
$$

\section{REFERENCES}

[1] R. Apéry, Sur les branches superlinéaires des courbes algébriques, C. R. Acad. Sci. Paris, 222 (1946). MR0017942(8:221a)

[2] V. Barucci, D. E. Dobbs and M. Fontana, "Maximality properties in numerical semigroups and applications to one-dimensional analytically irreducible local domains", Memoirs of the Amer. Math. Soc. 598 (1997). MR1357822 (97g:13039)

[3] J. Bertin and P. Carbone, Semi-groupes d'entiers et application aux branches, J. Algebra 49 (1987), 81-95. MR0568894 (58:27957)

[4] R. Fröberg, G. Gottlieb and R. Häggkvist, On numerical semigroups, Semigroup Forum 35 (1987), 63-83. MR0880351 (88d:20092)

[5] J. Herzog, Generators and relations of abelian semigroups and semigroup rings, Manuscripta Math., 3 (1970), 175-193. MR.0269762 (42:4657)

[6] E. Kunz, The value-semigroup of a one-dimensional Gorenstein ring, Proc. Amer. Math. Soc., 25 (1973), 748-751. MR0265353 (42:263)

[7] J. C. Rosales and P. A. García-Sánchez, "Finitely generated commutative monoids," Nova Science Publishers, New York, 1999. MR.1694173 (2000d:20074)

[8] J. C. Rosales, P. A. García-Sánchez and J. M. Urbano-Blanco, Modular Diophantine inequalities and numerical semigroups, Pacific J. Math. 218 (2005), 379-398.

[9] J. C. Rosales, P. A. García-Sánchez, J. I. García-García and J. M. Urbano-Blanco, Proportionally modular Diophantine inequalities, J. Number Theory 103 (2003), 281-294. MR 2020273 (2004k:20127)

Departamento de Álgebra, Universidad de Granada, E-18071 Granada, Spain

E-mail address: jrosales@ugr.es 